\title{
TGIF-DB: terse genomics interface for developing botany
}

\author{
Daisuke Tsugama* (i) and Tetsuo Takano
}

\begin{abstract}
Objectives: Pearl millet (Pennisetum glaucum) is a staple cereal crop for semi-arid regions. Its whole genome sequence and deduced putative gene sequences are available. However, the functions of many pearl millet genes are unknown. Situations are similar for other crop species such as garden asparagus (Asparagus officinalis), chickpea (Cicer arietinum) and Tartary buckwheat (Fagopyrum tataricum). The objective of the data presented here was to improve functional annotations of genes of pearl millet, garden asparagus, chickpea and Tartary buckwheat with gene annotations of model plants, to systematically provide such annotations as well as their sequences on a website, and thereby to promote genomics for those crops.

Data description: Sequences of genomes and transcripts of pearl millet, garden asparagus, chickpea and Tartary buckwheat were downloaded from a public database. These transcripts were associated with functional annotations of their Arabidopsis thaliana and rice (Oryza sativa) counterparts identified by BLASTX. Conserved domains in protein sequences of those species were identified by the HMMER scan with the Pfam database. The resulting data was deposited in the figshare repository and can be browsed on the Terse Genomics Interface for Developing Botany (TGIF-DB) website (http://webpark2116.sakura.ne.jp/rlgpr/).
\end{abstract}

Keywords: Arabidopsis, Garden asparagus, Chickpea, Genomics, Pearl millet, Plant, Rice, Tartary buckwheat

\section{Objective}

Pearl millet (Pennisetum glaucum) is a staple cereal crop for semi-arid regions. Its whole genome was sequenced and putative gene sequences were deduced [1]. Functions of some of the pearl millet genes have also been either examined by experiments or predicted on the basis of their homologies to specific, targeted gene sets with known functions ([2, 3]; for example). However, functional annotations of the pearl millet genes are neither sufficient nor systematic. Situations are similar in many other plant species such as garden asparagus (Asparagus officinalis), chickpea (Cicer arietinum) and Tartary buckwheat (Fagopyrum tataricum) [4-6 respectively, for analyses of their genomes]. Arabidopsis thaliana and

*Correspondence: dtsugama@anesc.u-tokyo.ac.jp Asian Natural Environmental Science Center (ANESC), The University of Tokyo, 1-1-1 Midori-cho, Nishi-tokyo-shi, Tokyo 188-0002, Japan rice (Oryza sativa) are dicot and monocot model species, respectively, and have better functional annotations for each gene ([7, 8]; for example). The objective of the data presented here was to improve the functional annotations of genes of pearl millet by systematic homology searches with databases for Arabidopsis genes, rice genes and protein conserved domains, to develop a platform for browsing the resulting data, and thereby to promote pearl millet genomics.

\section{Data description}

The whole genome sequences and transcript (or protein coding) sequences that were deduced from the genome sequences of pearl millet, garden asparagus, chickpea and Tartary buckwheat as well as genome annotation files in the general feature format (GFF) were downloaded from the International Pearl Millet Genome 
Table 1 Overview of data files/data sets

\begin{tabular}{|c|c|c|c|}
\hline Label & Name of data file/data set & File types (file extension) & Data repository and identifier (DOI or accession number) \\
\hline Data set 1 & Ao_gene_seqs.fa & Fasta (.fa) file & Figshare (https://doi.org/10.6084/m9.figshare.13565168.v2) [16] \\
\hline Data set 2 & Ao_promoter_seqs.fa & Fasta (.fa) file & Figshare (https://doi.org/10.6084/m9.figshare.13565168.v2) [16] \\
\hline Data set 3 & Ao_protein_seqs.fa & Fasta (.fa) file & Figshare (https://doi.org/10.6084/m9.figshare.13565168.v2) [16] \\
\hline Data set 4 & Ao_proteins_At_proteins_blastp.txt & Text (.txt) file & Figshare (https://doi.org/10.6084/m9.figshare.13565168.v2) [16] \\
\hline Data set 5 & Ao_proteins_ELM.txt & Text (.txt) file & Figshare (https://doi.org/10.6084/m9.figshare.13565168.v2) [16] \\
\hline Data set 6 & Ao_proteins_Os_proteins_blastp.txt & Text (.txt) file & Figshare (https://doi.org/10.6084/m9.figshare.13565168.v2) [16] \\
\hline Data set 7 & Ao_proteins_Pfam.txt & Text (.txt) file & Figshare (https://doi.org/10.6084/m9.figshare.13565168.v2) [16] \\
\hline Data set 8 & Ao_transcript_seqs.fa & Fasta (.fa) file & Figshare (https://doi.org/10.6084/m9.figshare.13565168.v2) [16] \\
\hline Data set 9 & Ao_transcripts_hairpin_miRNA_blastn.txt & Text (.txt) file & Figshare (https://doi.org/10.6084/m9.figshare.13565168.v2) [16] \\
\hline Data set 10 & Ca_gene_seqs.fa & Fasta (.fa) file & Figshare (https://doi.org/10.6084/m9.figshare.13565168.v2) [16] \\
\hline Data set 11 & Ca_promoter_seqs.fa & Fasta (.fa) file & Figshare (https://doi.org/10.6084/m9.figshare.13565168.v2) [16] \\
\hline Data set 12 & Ca_protein_seqs.fa & Fasta (.fa) file & Figshare (https://doi.org/10.6084/m9.figshare.13565168.v2) [16] \\
\hline Data set 13 & Ca_proteins_At_proteins_blastp.txt & Text (.txt) file & Figshare (https://doi.org/10.6084/m9.figshare.13565168.v2) [16] \\
\hline Data set 14 & Ca_proteins_ELM.txt & Text (.txt) file & Figshare (https://doi.org/10.6084/m9.figshare.13565168.v2) [16] \\
\hline Data set 15 & Ca_proteins_Os_proteins_blastp.txt & Text (.txt) file & Figshare (https://doi.org/10.6084/m9.figshare.13565168.v2) [16] \\
\hline Data set 16 & Ca_proteins_Pfam.txt & Text (.txt) file & Figshare (https://doi.org/10.6084/m9.figshare.13565168.v2) [16] \\
\hline Data set 17 & Ca_transcript_seqs.fa & Fasta (.fa) file & Figshare (https://doi.org/10.6084/m9.figshare.13565168.v2) [16] \\
\hline Data set 18 & Ft_gene_seqs.fa & Fasta (.fa) file & Figshare (https://doi.org/10.6084/m9.figshare.13565168.v2) [16] \\
\hline Data set 19 & Ft_promoter_seqs.fa & Fasta (.fa) file & Figshare (https://doi.org/10.6084/m9.figshare.13565168.v2) [16] \\
\hline Data set 20 & Ft_protein_seqs.fa & Fasta (.fa) file & Figshare (https://doi.org/10.6084/m9.figshare.13565168.v2) [16] \\
\hline Data set 21 & Ft_proteins_At_proteins_blastp.txt & Text (.txt) file & Figshare (https://doi.org/10.6084/m9.figshare.13565168.v2) [16] \\
\hline Data set 22 & Ft_cds_seqs.fa & Fasta (.fa) file & Figshare (https://doi.org/10.6084/m9.figshare.13565168.v2) [16] \\
\hline Data set 23 & Ft_proteins_Os_proteins_blastp.txt & Text (.txt) file & Figshare (https://doi.org/10.6084/m9.figshare.13565168.v2) [16] \\
\hline Data set 24 & Ft_proteins_Pfam.txt & Text (.txt) file & Figshare (https://doi.org/10.6084/m9.figshare.13565168.v2) [16] \\
\hline Data set 25 & Ft_transcript_seqs.fa & Fasta (.fa) file & Figshare (https://doi.org/10.6084/m9.figshare.13565168.v2) [16] \\
\hline Data set 26 & Pg_gene_segs.fa & Fasta (.fa) file & Figshare (https://doi.org/10.6084/m9.figshare.13565168.v2) [16] \\
\hline Data set 27 & Pg_promoter_seqs.fa & Fasta (.fa) file & Figshare (https://doi.org/10.6084/m9.figshare.13565168.v2) [16] \\
\hline Data set 28 & Pg_protein_segs.fa & Fasta (.fa) file & Figshare (https://doi.org/10.6084/m9.figshare.13565168.v2) [16] \\
\hline Data set 29 & Pg_proteins_At_proteins_blastp.txt & Text (.txt) file & Figshare (https://doi.org/10.6084/m9.figshare.13565168.v2) [16] \\
\hline Data set 30 & Pg_proteins_ELM.txt & Text (.txt) file & Figshare (https://doi.org/10.6084/m9.figshare.13565168.v2) [16] \\
\hline Data set 31 & Pg_proteins_Os_proteins_blastp.txt & Text (.txt) file & Figshare (https://doi.org/10.6084/m9.figshare.13565168.v2) [16] \\
\hline Data set 32 & Pg_proteins_Pfam.txt & Text (.txt) file & Figshare (https://doi.org/10.6084/m9.figshare.13565168.v2) [16] \\
\hline Data set 33 & Pg_transcript_segs.fa & Fasta (.fa) file & Figshare (https://doi.org/10.6084/m9.figshare.13565168.v2) [16] \\
\hline Data set 34 & Pg_transcripts_hairpin_miRNA_blastn.txt & Text (.txt) file & Figshare (https://doi.org/10.6084/m9.figshare.13565168.v2) [16] \\
\hline Data file 1 & Figure_TGIF-DB_snapshots.tif & Figure (.tif) file & Figshare (https://doi.org/10.6084/m9.figshare.13565168.v2) [16] \\
\hline
\end{tabular}

Sequencing Consortium (IPMGSC) website [9], the Asparagus Genome Project website [10], the National Center for Biotechnology Information (NCBI) Chickpea Genome website (with Genome ID 2992) [11] and the MBKBASE Tartary Buckwheat Genome Project website [12], respectively. The sequences and functional annotations of Arabidopsis proteins (TAIR10 versions) were downloaded from The Arabidopsis Information Resource (TAIR) website [7], and those of rice (RGAP 7 versions) were downloaded from the Rice Genome Annotation Project (RGAP) website [8]. BLASTX on the BLAST + suite [13] was performed with the transcript sequences of those crop species as queries and with either the Arabidopsis protein sequences or rice protein sequences as the database. The threshold E-value was set as $1 \mathrm{e}-20$, which is more stringent than the default value (10.0), for this analysis. The transcripts (or genes) of the crop species were then associated with the functional annotations of the corresponding Arabidopsis and rice proteins identified by the BLASTX search. Protein sequences of pearl millet, garden asparagus, chickpea and Tartary buckwheat were deduced from their transcript sequences, and the Pfam database [14] was searched by the hmmscan program for HMMER (version 3.3) [15] to 
identify conserved domains in those proteins. The threshold E-value was set as $1 \mathrm{e}-5$, which is more stringent than the default value (10), for this analysis. A genomic locus sequence, which consists of exons and introns, and a promoter sequence, which is a 3-kb upstream sequence from the start codon, for each gene were extracted on the basis of the whole genome sequences and the GFF files. The resulting data for the gene sequences and their functional annotations for pearl millet, garden asparagus, chickpea and Tartary buckwheat were deposited in the figshare repository (Data sets 1-34 in Table 1) [16]. A website, Terse Genomics Interface for Developing Botany (TGIF-DB), was developed to browse these data [17] (see Data file 1 in Table 1 for a TGIF-DB interface). The programs in the BLAST+ suite [13] and the genome browser JBrowse [18] were included as a part of TGIF-DB.

\section{Limitations}

- Some proteins of the species used do not appear to have conserved domains and/or any close homolog in either Arabidopsis or rice.

- Some pearl millet genes have been characterized by targeted analyses ([2,3]; for example) but information about such analyses has not been included in the data set presented.

\section{Abbreviation}

GFF: General feature format.

\section{Acknowledgements}

The authors greatly appreciate data and advice from Dr. Shashi Kumar Gupta and his colleagues in International Crops Research Institute for the Semi-Arid Tropics (ICRISAT). The authors thank their colleagues to test former versions of TGIF-DB.

\section{Authors' contributions}

DT and TT collected data, analysed the data, made TGIF-DB and wrote the manuscript. Both authors read and approved the final manuscript.

\section{Funding}

This work was supported by JSPS (Japan Society for the Promotion of Science) Kakenhi Grant (Grant Number: 19KK0155 and 19K15827).

\section{Availability of data and materials}

The datasets generated during and/or analysed during the current study are available in the figshare repository, https://doi.org/10.6084/m9.figshare.13565 168.v2 [16]. The data can be browsed on the TGIF-DB website, http://webpa rk2116.sakura.ne.jp/rlgpr/ [17]. Please see Table 1 and references [10, 11] for details and links to the data.

\section{Declarations}

Ethics approval and consent to participate Not applicable.
Consent for publication

Not applicable.

\section{Competing interests}

The authors declare that they have no competing interests.

Received: 14 January 2021 Accepted: 5 May 2021

Published online: 13 May 2021

\section{References}

1. Varshney RK, Shi C, Thudi M, Mariac C, Wallace J, Qi P, et al. Pearl millet genome sequence provides a resource to improve agronomic traits in arid environments. Nat Biotechnol. 2017;35:969-76.

2. Shinde H, Dudhate A, Tsugama D, Gupta SK, Liu S, Takano T. Pearl millet stress-responsive NAC transcription factor PgNAC21 enhances salinity stress tolerance in Arabidopsis. Plant Physiol Biochem. 2019;135:546-53.

3. Chanwala J, Satpati S, Dixit A, Parida A, Giri MK, Dey N. Genome-wide identification and expression analysis of WRKY transcription factors in pearl millet (Pennisetum glaucum) under dehydration and salinity stress. BMC Genomics. 2020;21:231.

4. Harkess A, Zhou J, Xu C, Bowers JE, Van der Hulst R, Ayyampalayam S, et al. The asparagus genome sheds light on the origin and evolution of a young Y chromosome. Nat Commun. 2017:8:1279.

5. Varshney RK, Song C, Saxena RK, Azam S, Yu S, Sharpe AG, et al. Draft genome sequence of chickpea (Cicer arietinum) provides a resource for trait improvement. Nat Biotechnol. 2013;31:240-6.

6. Zhang L, Li X, Ma B, Gao Q, Du H, Han Y, et al. The Tartary buckwheat genome provides insights into rutin biosynthesis and abiotic stress tolerance. Mol Plant. 2017:10:1224-37.

7. Berardini TZ, Reiser L, Li D, Mezheritsky Y, Muller R, Strait E, Huala E. The Arabidopsis information resource: making and mining the "gold standard" annotated reference plant genome. Genesis. 2015;53:474-85.

8. Kawahara Y, de la Bastide M, Hamilton JP, Kanamori H, McCombie WR, Ouyang S, et al. Improvement of the Oryza sativa Nipponbare reference genome using next generation sequence and optical map data. Rice. 2013;6:4.

9. Varshney RK, Shi C, Thudi M, Mariac C, Wallace J, Qi P, et al. International pearl millet genome sequencing consortium (IPMGSC). 2021. http:// cegsb.icrisat.org/ipmgsc. Accessed 14 Jan 2021.

10. Asparagus Genome Project. 2021. http://asparagus.uga.edu/tripal. Accessed 16 Mar 2021.

11. NCBI genome for Cicer arietinum (chickpea). 2021. https://www.ncbi.nlm. nih.gov/genome/2992. Accessed 16 Mar 2021.

12. MBKBASE, introduction to tartary buckwheat genome project. 2021. http://mbkbase.org/Pinku1. Accessed 16 Mar 2021.

13. Camacho C, Coulouris G, Avagyan V, Ma N, Papadopoulos J, Bealer K, Madden TL. BLAST+: architecture and applications. BMC Bioinform. 2009;10:421.

14. El-Gebali S, Mistry J, Bateman A, Eddy SR, Luciani A, Potter SC, et al. The Pfam protein families database in 2019. Nucleic Acids Res. 2019;47:D427-32.

15. Mistry J, Finn RD, Eddy SR, Bateman A, Punta M. Challenges in homology search: HMMER3 and convergent evolution of coiled-coil regions. Nucleic Acids Res. 2013;41:e121.

16. Tsugama D. TGIF-DB datasets. figshare. 2021. https://doi.org/10.6084/m9. figshare.13565168.v5.

17. Tsugama D. TGIF-DB. http://webpark2116.sakura.ne.jp/rlgpr. Accessed 14 Jan 2021.

18. Buels R, Yao E, Diesh CM, Hayes RD, Munoz-Torres M, Helt G, et al. JBrowse: a dynamic web platform for genome visualization and analysis. Genome Biol. 2016;17:66.

\section{Publisher's Note}

Springer Nature remains neutral with regard to jurisdictional claims in published maps and institutional affiliations. 